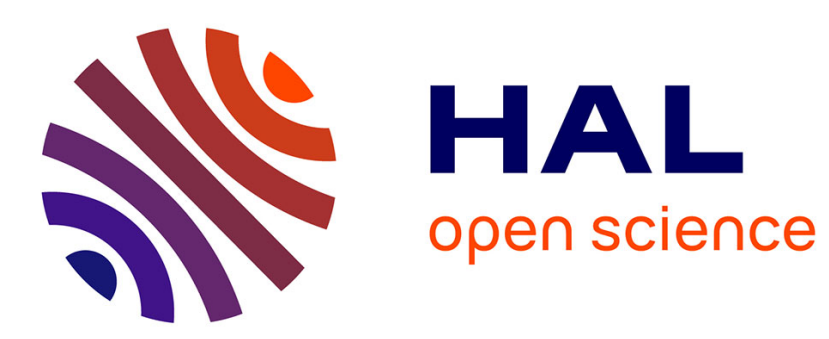

\title{
Magnetismus und lokales Molekularfeld (Nobel-Vortrag)
}

Louis Néel

\section{To cite this version:}

Louis Néel. Magnetismus und lokales Molekularfeld (Nobel-Vortrag). Angewandte Chemie, 1971, 83 (21), pp.838-848. 10.1002/ange.19710832106 . hal-02888367

\section{HAL Id: hal-02888367 https://hal.science/hal-02888367}

Submitted on 29 Jul 2020

HAL is a multi-disciplinary open access archive for the deposit and dissemination of scientific research documents, whether they are published or not. The documents may come from teaching and research institutions in France or abroad, or from public or private research centers.
L'archive ouverte pluridisciplinaire HAL, est destinée au dépôt et à la diffusion de documents scientifiques de niveau recherche, publiés ou non, émanant des établissements d'enseignement et de recherche français ou étrangers, des laboratoires publics ou privés. 


\title{
Magnetismus und lokales Molekularfeld (Nobel-Vortrag) ${ }^{[* *]}$
}

\author{
Von Louis Néel ${ }^{[*]}$
}

\section{Molekulares Feld nach Weiss}

Es ist seit langem bekannt, daß der Ferromagnetismus auf Wechselwirkungen zwischen atomaren magnetischen Momenten beruht, die trotz der thermischen Bewegung eine parallele Ausrichtung der Atommagnete untereinander zur Folge haben. Zur quantitativen Deutung der experimentellen Befunde ging Pierre Weiss ${ }^{[1]}$ von der Hypothese aus, daß sich ein ferromagnetischer Stoff ebenso verhält wie ein rein paramagnetischer als Träger unabhängiger Dipolmomente, dessen Magnetisierung gegeben ist durch

$J=f\left(\frac{H}{T}\right)$

und daß die maßgebenden Wechselwirkungen einem fiktiven magnetischen Feld $h_{m}$ gleichkommen, dem sogenannten molekularen Feld, das Weiss der Magnetisierung $J$ proportional setzte:

$\mathrm{h}_{\mathrm{m}}=\mathrm{nJ}$

Dieses Molekularfeld $h_{m}$ wirkt außer einem äußeren Feld $H$. Für hinreichend schwaches $H$ erhält man auf diesem Wege das Curie-Weiss-Gesetz der Magnetisierung:

$J=\frac{C H}{T-\Theta}$ mit $\Theta=n C$

Ist $\Theta$ positiv, so wird die Suszeptibilität $\mathrm{J} / \mathrm{H}$ unendlich groß, wenn die Temperatur T bis zur Curie-Temperatur $\Theta$ absinkt. Zwischen $\Theta$ und dem absoluten Nullpunkt der Temperatur hat der betrachtete Stoff unter dem Einfluß seines Molekularfeldes $h_{m}=n J_{s}$ eine gewisse spontane Magnetisierung $\mathrm{J}_{\mathrm{s}}$.

In diesem Bild betrachtet man das Molekularfeld ais ein homogenes Feld im Innern des ferromagnetischen Stoffes. Um jedoch seine Theorie zu vervollständigen, definierte Weiss unter Einbeziehung der inneren Energie U ein energetisches Molekularfeld

$\mathrm{H}_{\mathrm{r}}=-\frac{\hat{c} \mathrm{U}}{\hat{c} \mathrm{~J}}$

sowie ein korrektives Molekularfeld $\mathrm{h}_{\mathrm{m}}$, das entsprechend Gleichung (1) aus

$J=f\left(\frac{H+h_{m}}{T}\right)$

hervorgeht.

[*] Prof. Dr. L. Néel

Laboratoire d'Électrostatique et de Physique du Métal CNRS

F-38 Grenoble, Rue des Martyrs (Frankreich)

[**] Copyright $(0$ The Nobel Foundation 1971. - Wir danken der Nobel-Stiftung, Stockholm, für die Genehmigung zum Druck dieser Ubersetzung. - Die Schreibweise der Gleichungen sowie die Symbole für physikalische Größen wurden vom französischen Originaltext unverändert übernommen.
Eine thermodynamische Betrachtung liefert als Verbindung dieser beiden Feldgrößen die Beziehung

$H_{m}=h_{m}-T \frac{d h_{m}}{d T}$

Dieser gedanklich sehr befriedigende Deutungsweg ermöglicht es, dic energetischen Eigenschaften ferromagnetischer Stoffe in eleganter und einfacher Weise zu erklären. Er hat allerdings den Nachteil, daß die Homogenität des Molekularfeldes wie ein Dogma eingeführt wird, ebenso wie alles was daraus folgt, insbesondere die lineare Beziehung zwischen der reziproken Suszeptibilität und der Temperatur oberhalb der Curie-Temperatur. Dieser Umstand hatte die Weiterentwicklung der Theorie früher erheblich verzögert.

\section{Das lokale Molekularfeld}

Andererseits konnte Weiss keine befriedigende Erklärung für den Ursprung des Molekularfeldes geben. Erst 1928 fand Heisenberg theoretisch einen möglichen Wechselwirkungsmechanismus in passender Größenordnung. Für unsere Zwecke genügt hier der Hinweis, daß es sich um Wechselwirkungen auf sehr kurze Entfernung handelt, die weitgehend beschränkt sind auf die nächsten Nachbarn im Kristallgitter, jedoch vernachlässigbar klein bleiben für zweit- und drittnächste Nachbarn.

Wenn man demnach eine Legierung betrachtet, die aus zwei Atomarten A und B in unregelmäßiger Verteilung besteht, so müssen sich die Umgebungen einzelner Atome voneinander stark unterscheiden; die Hypothese eines überall gleichen Molekularfeldes an den Wirkungsorten der verschiedenen Umgebungen kann so nur eine sehr schlechte Annäherung an den wirklichen Zustand sein. Das Problem einer strengen Berechnung derartiger verschiedener Wechselwirkungen ist heute von einer Lösung noch weit entfernt. Aber auch unter Beibehaltung einfacher theoretischer Annahmen im Sinne des Molekularfeldes ist ein wesentlicher Fortschritt möglich, wenn man das hinzunimmt, was ich "lokale Molekularfelder" genannt habe.

Die Hypothese von Weiss bleibt erhalten, sofern man die Energie $E_{c}$ des Systems der Atome A und B in der Form

$\mathrm{E}_{\mathrm{c}}=-\frac{1}{2} \mathrm{n}\left(\mathrm{J}_{\mathrm{A}}+\mathrm{J}_{\mathrm{B}}\right)^{2}$

schreibt, wobei $\mathrm{J}_{\mathrm{A}}$ und $\mathrm{J}_{\mathrm{B}}$ die Magnetisierungen der Atome A bzw. $B$ bedeuten. Tatsächlich ist diese Energie jedoch die Summe verschiedener Beiträge der nächstbenachbarten Atompaare A-A, A-B, B-B. Daher muß man besser schreiben:

$E_{c}=-\frac{1}{2}\left(n_{A A} J_{\AA}^{2}+2 n_{A B} J_{A} J_{B}+n_{B B} J_{B}^{2}\right)$ 
Unter Aufgabe des Modells eines homogenen Molekularfeldes erhält man die lokalen Molekularfelder $\mathrm{h}_{\mathrm{A}}=\mathrm{n}_{\mathrm{AA}} \mathrm{J}_{\mathrm{A}}+\mathrm{n}_{\mathrm{AB}} \mathrm{J}_{\mathbf{B}}$ und $\mathrm{h}_{\mathrm{B}}=\mathrm{n}_{\mathrm{AB}} \mathrm{J}_{\mathrm{A}}+\mathrm{n}_{\mathrm{BB}} \mathrm{J}_{\mathbf{B}}$, die jeweils an den Orten der Atomarten $A$ und $B$ wirksam sind.

Diese Betrachtungsweise entwickelte ich erstmalig im Jahre $1932^{[2]}$. Ich zeigte damals, daß die Suszeptibilität $\chi$ einer Legierung aus $\mathrm{A}$ und $\mathrm{B}$ im Atomzahlverhältnis $\mathrm{P}: \mathrm{Q}$ mit Curie-Konstanten $C_{A}$ und $C_{B}$ sich darstellen läßt durch

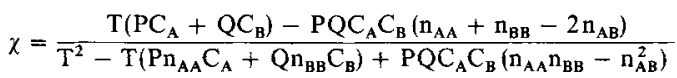

Anstatt einer Geraden folgt der Kehrwert $1 / \chi$ der Suszeptibilität in Abhängigkeit von der Temperatur nunmehr einer Hyperbel.

Ich benutzte diese Theorie zur Deutung von Eigenschaften der Platin-Kobait-Legierungen und etwas später auch der Eisen-Kobalt-, Eisen-Nickel- und Kobalt-Nickel-Legierungen ${ }^{[3]}$.

Zur damaligen Zeit wurde eine solche Interpretationsmöglichkeit wenig günstig aufgenommen. Die Existenz einer Geraden in der Darstellung $(1 / \chi, T)$ nach Curie-Weiss war ein festgefahrenes Dogma. Wenn die Experimente krumme Kurven ergaben, zog man es oft vor, diese Kurven aus aneinandergesetzten geraden Stücken zusammenzuflicken, welche jeweils einem magnetischen Zustand entsprechen sollten, der dem Curie-Weiss-Gesetz gehorcht.

\section{Die Fluktuationen des Weiss'schen Molekularfeldes}

In der primitiven Theorie von Weiss haben sowohl der Koeffizient $\mathbf{n}$ des Molekularfeldes als auch die Curie-Temperatur $\Theta$ positive Werte, und man versteht sehr gut, daß dieses Molekularfeld einen Ordnungszustand bewirkt, bei dem alle atomaren Momente gleichsinnig parallel gerichtet sind. Aber Weiss und seine Mitarbeiter stellten bald fest, daß die paramagnetischen Eigenschaften gewisser Salze sich entsprechend einer Gleichung vom Typ (3) verhalten, allerdings mit einem negativen Wert der Konstante $\Theta$, d. h. mit einem negativen Molekularfeld. Es ist nicht einzusehen, wie ein solches Feld bei tiefer Temperatur einen geordneten Zustand hervorrufen könnte.

Außerdem versagte die Theorie von Weiss für die Erklärung der Eigenschaften paramagnetischer Metalle wie Mangan und Chrom, deren Suszeptibilität von der Temperatur nahezu unabhängig und überdies viel zu groß für einen paramagnetischen Stoff ist, dessen Eigenschaften nach Pauli von Elektronen in einem Energieband herrühren.

Ungefähr dieser Zustand der Probleme lag 1930 vor, als ich mich für die beiden verschiedenen Curie-Temperaturen interessierte, d.h. für die Beobachtung, daß die aufgrund des Curie-Weiss-Gesetzes gemessene „paramagnetische Curie-Temperatur“ $\Theta_{p}$ nicht übereinstimmt mit der „ferromagnetischen Curie-Temperatur" $\Theta_{f}$, bei der die spontane Magnetisierung verschwindet, während die Theorie von Weiss $\Theta_{\mathrm{p}}=\Theta_{\mathrm{r}}$ fordert. Eine Erklärung dieses Widerspruchs suchte ich in den thermischen Schwankungen des Moleku- larfeldes, deren Existenz nicht zweifelhaft war, da ja dieses Feld von der Wechselwirkung benachbarter Atome abhängt. Es handelt sich dabei um zeitliche Schwankungen. Aber es lag für mich von vornherein nahe, auch an die räumlichen Schwankungen zu denken und insbesondere Schlußfolgerungen für das elementare Gesetz der magnetischen Wechselwirkungen zu ziehen anhand der Existenz einer Kopplungsenergie $w \cdot \cos \alpha$ zwischen zwei nächstbenachbarten Atomen, wobei $\alpha$ den Winkel zwischen deren magnetischen Momenten bedeutet.

\section{Die paramagnetische Konstante}

Die Konstante w kann negativ oder positiv sein - negativ im Falle des Ferromagnetismus, positiv in dem des negativen Molekularfeldes. In diesem zweiten Fall ist die Konzeption des Molekularfeldes zwar für hohe Temperaturen anwendbar, für die der Zustand aller Atome im Mittel identisch ist, nicht jedoch für tiefe Temperaturen, bei denen die atomaren magnetischen Dipole eine Gruppierung zu Paaren antiparalleler Momente bevorzugen.

Aus diesem Grunde kam ich zur Hypothese (vgl. ${ }^{[2]}$, S. 64), $\mathrm{da} \beta$ ein raumzentriertes, kubisches Gitter im stabilen Gleichgewicht bei tiefer Temperatur aus zwei ineinandergeschachtelten, einfachen kubischen Gittern bestehen kann, deren jeweilige Magnetisierung zueinander antiparallel gerichtet ist, so wie es Abbildung 1 für ein ebenes Gitter andeutet. Diese Anordnung deformiert sich unter der Einwirkung eines äußeren Feldes (Abb. 2), so daß im Mittel je Atom die Magnetisierung

$\bar{\mu}=\frac{\mu^{2} \mathrm{H}}{6 \mathrm{pw}}$

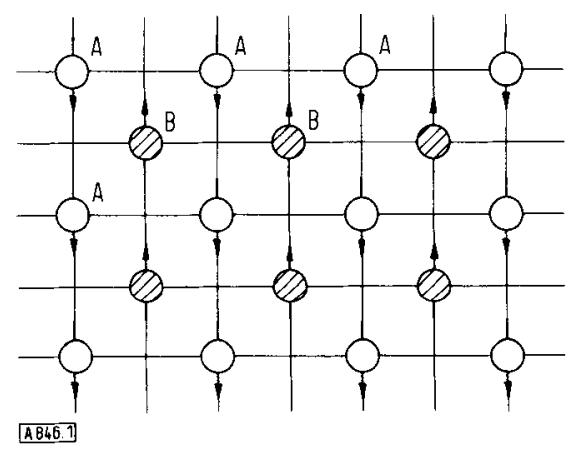

Abb. 1. Zerlegung eines ebenen Gitters in zwei antiparallel magnetisierte Teilgitter.

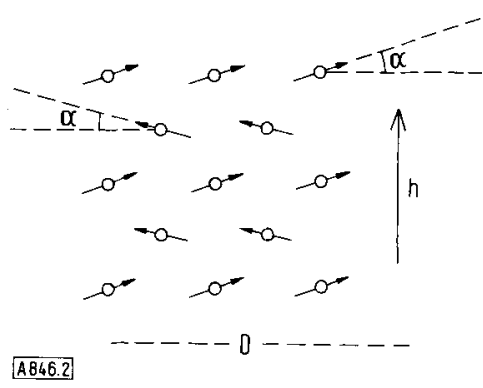

Abb. 2. Deformation einer regelmäßigen Anordnung antiparalleler Atommagnete unter dem Einfluß eines Querfeldes $h$. 
auftritt, wenn $\mu$ das atomare Moment und 2 p die Zahl der Nachbarn bedeuten. Man erhält so einen konstanten Paramagnetismus, d.h. eine Suszeptibilität, die nicht von der Feldstärke und nicht von der Temperatur abhängt.

Bei hoher Temperatur jedoch muß man wieder ein Gesetz nach Curie-Weiss vom Typ (3) erwarten.

Um einen Ansatz für den Übergang vom konstanten Paramagnetismus zum Paramagnetismus nach Curie-Weiss zu finden, nahm ich vereinfachend an, daß man die Atome zu voneinander unabhängigen Paaren gruppieren kann und die Kopplungsenergie je Paar w $\cdot \cos \alpha$ beträgt. Hieraus folgt

$\bar{\mu}=\frac{\mu^{2} \mathrm{H}}{3 \mathrm{w}}\left(1-\frac{2 \mathrm{w}}{\mathrm{kT}} \cdot \frac{1}{\mathrm{e}^{2 \mathrm{w} / \mathrm{k}} \mathrm{T}}-\overline{1}\right)$

Abbildung 3 zeigt den Kehrwert der Suszeptibilität in Abhängigkeit von der Temperatur. Zwischen $\mathrm{T}=0$ und $\mathrm{T}=\Theta$ ändert sich die Suszeptibilität nur um $1.4 \%$.

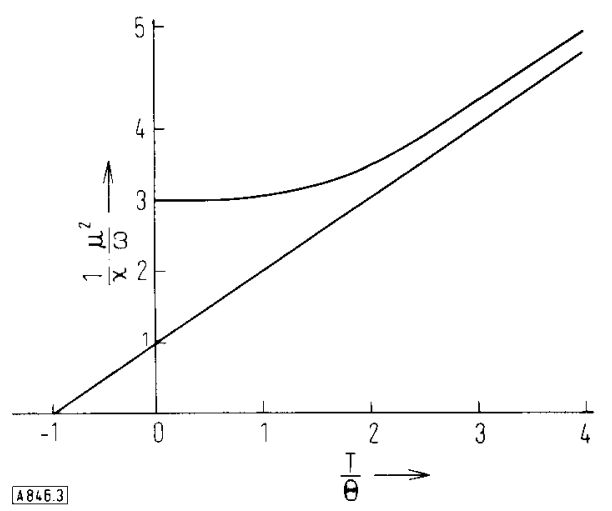

Abb. 3. Temperaturfunktion des Kehrwertes der Suszeptibilität einer Anordnung antiparallel magnetisierter Atompaare mit unregelmäßiger statistischer Orientierung.

In einer anderen Abhandlung, fast um die gleiche Zeit, schlug ich vor ${ }^{[4]}$, den konstanten Paramagnetismus des Mangans und des Chroms durch den soeben beschriebenen Mechanismus zu erklären und dafür die Beträge $\Theta=-1720^{\circ} \mathrm{K}$ bzw. $-4150^{\circ} \mathrm{K}$ anzusetzen. Als Stütze dieser theoretischen Deutung konnte ich nachweisen, daß bei wachsendem Zusatz von Kupfer, Silber oder Gold zum Mangan und zum Chrom, also durch "Verdünnen" des reinen Ausgangsstoffes, ein fortschreitender Übergang vom konstanten Paramagnetismus zum Paramagnetismus nach Curie-Weiss erkennbar wird, ganz so, wie man es nach der hier skizzierten Theorie erwarten müßte, wenn die Wechselwirkungskräfte, d. h. auch $\Theta$, herabgesetzt werden.

Im Jahre 1936 beschäftigte ich mich wiederum mit der Theorie der magnetischen Kopplungskonstante im Bereich tiefer Temperaturen, und zwar unter Berücksichtigung einer Kopplung der atomaren Momente mit dem Kristallgitter ${ }^{[5]}$. Für die Kopplungsenergie wurde $w^{\prime \prime}=\cos 2 \delta$ angesetzt. Darin bedeutet $\delta$ den Winkel zwischen dem atomaren Moment und einer energetisch bevorzugten Richtung im Kristallgitter. Es zeigt sich, daß die magnetische Suszeptibilität eine recht komplizierte Funktion des magne- tischen Feldes $\mathrm{H}$ und des Winkels $\beta$ zwischen $\mathrm{H}$ und $\mathrm{D}$ ist. In Abbildung 4 ist diese Funktion für verschiedene Beträge $\beta$ in Stufen von je $10^{\circ}$ dargestellt.

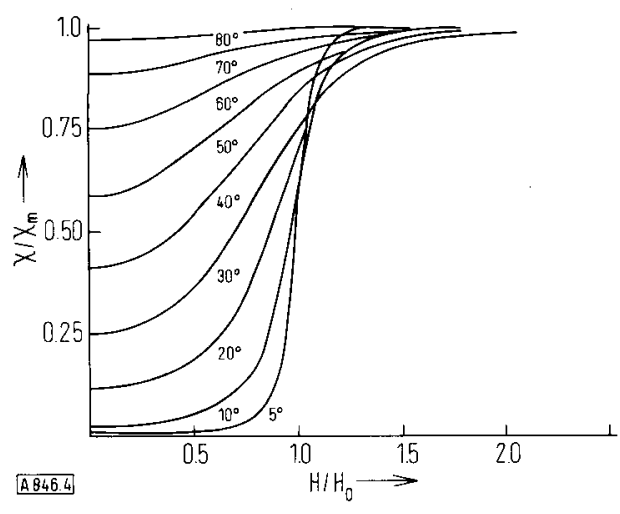

Abb. 4. Suszeptibilität eines Raumgitters antiparalleler magnetischer Momente als Funktion eines äußeren magnetischen Feldes. Parameter: Winkel zwischen Feld und magnetischer Vorzugslage im Kristallgitter.

Wirkt das Feld H speziell parallel zur Vorzugslage D, so bleibt die Suszeptibilität bei schwacher Feldstärke zunächst Null und springt dann bei der Feldstärke

$$
\mathrm{H}=\mathrm{H}_{0}=\left(\frac{8 \mathrm{pw} \mathrm{w}^{\prime \prime}}{\mu^{2}}\right)^{1 / 2}
$$

diskontinuierlich auf $\chi_{\mathrm{m}}=\mu^{2} / 4 \mathrm{pw}$, unabhängig von $\mathrm{H}$. Wenn nämlich $\mathrm{H}$ den Betrag $\mathrm{H}_{0}$ erreicht, orientieren sich die atomaren Momente, die anfänglich parallel zu D und $H$ lagen, plötzlich quer zu D und H. Fünfzehn Jahre später, im Jahre 1951, beobachteten C. J. Gorter und Mitarbeiter ${ }^{[6]}$ erstmalig dieses Phänomen an $\mathrm{CuCl}_{2} \cdot 2 \mathrm{H}_{2} \mathrm{O}$ bei $4.1^{\circ} \mathrm{K}$.

Aus dieser Theorie folgt für einen polykristallinen Stoff, dessen Vorzugslagen D statistisch unregelmäßig verteilt sind, daß die Suszeptibilität in schwachen Feldern viel klei-

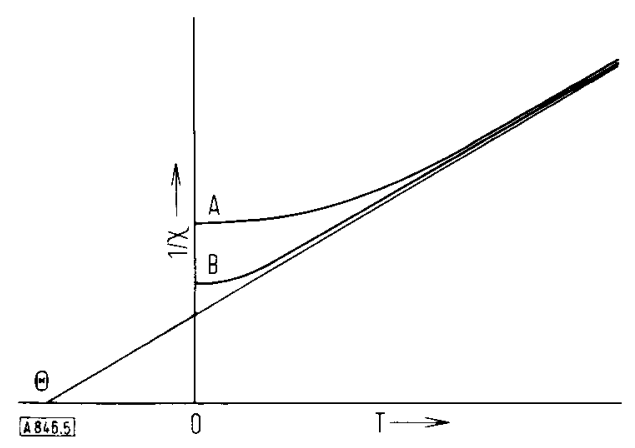

Abb. 5. Kehrwert der Suszeptibilität einer statistisch unregelmäßigen (polykristallinen) Mischung antiparalleler Atommagnete als Funktion der Temperatur und der Stärke des äußeren Feldes.

ner ist als in starken Feldern und von der Temperatur so abhängt, wie es Abbildung 5 schematisch zeigt. Wie man sieht, gibt es keine Übergangstemperatur: Der konstante Paramagnetismus geht völlig kontinuierlich über in den Paramagnetismus nach Curie-Weiss. 


\section{Entdeckung der antiferromagnetischen Übergangstemperatur}

Ebenfalls im Jahre 1936 hatte ich die Idee ${ }^{[5]}$, auf zwei Teilgitter $A$ und $B$ mit den Magnetisierungen $\mathrm{J}_{\mathrm{A}}$ und $\mathrm{J}_{\mathrm{B}}$, die ich schon in früheren Arbeiten benutzt hatte, die Theorie des lokalen Molekularfeldes anzuwenden und die Wechselwirkungen dabei durch fiktive Felder $\mathrm{H}_{\mathrm{A}}$ und $\mathrm{H}_{\mathrm{B}}$ darzustellen, wobei für tiefe Temperatur und $\mathrm{H}=0$ die fundamentale Beziehung $\mathrm{J}_{\mathrm{B}}=-\mathrm{J}_{\mathrm{A}}$ gelten soll.

Somit nehmen die beiden Teilgitter spontane Magnetisierungen in gegenläufiger Richtung an. Diese verschwinden bei einer gewissen Übergangstemperatur, die man heute auf Vorschlag von $C . J$. Gorter Néel-Temperatur nennt. Man hatte damals einen neuartigen magnetischen Stoff vor sich, der aus zwei identischen ferromagnetischen Stoffen besteht, deren spontane Magnetisierungen zueinander antiparallel gerichtet sind. Physikalische Effekte, die vom Quadrat der spontanen Magnetisierung abhängen, u.a. die Anomalie der spezifischen Wärme, müssen daher gleichartig auftreten wie in ferromagnetischen Stoffen.

Bei Abwesenheit einer Kopplung mit dem Kristallgitter bleibt die Suszeptibilität im Bereich $0<\mathrm{T}<\Theta_{\mathrm{N}}$ konstant und folgt für $\mathrm{T}>\Theta_{\mathrm{N}}$ einem Gesetz nach Curie-Weiss, ohne bei der Curie-Temperatur eine Diskontinuität zu zeigen.

Zwei Jahre später entdeckten Squire, Bizette und Tsaï ${ }^{[8]}$, $\mathrm{da} \beta \mathrm{MnO}$ diese vorhergesagten Eigenschaften besitzt, insbesondere eine Úbergangstemperatur bei $\Theta_{\mathrm{N}}=116^{\circ} \mathrm{K}$. Viel später erst, im Jahre 1949, bestätigten C. G. Shull und $S . J . S m a r t^{[9]}$ durch Neutronenbeugung, daß die atomaren Momente tatsächlich die antiparallele Orientierung aufweisen, die aufgrund der Theorie der zwei Teilgitter vorausgesagt worden war.

$F$. Bitter vervollständigte dann die Theorie ${ }^{[10]}$ durch Berechnung der Suszeptibilität bis zur Übergangstemperatur $\Theta_{N}$ für ein den gegenläufigen atomaren Momenten paralleles Magnetfeld. Er benutzte für diese neue Art magnetischer Stoffe zum ersten Male die Bezeichnung, antiferromagnetisch“. J. H. van Vleck ${ }^{[11]}$ trug 1941 eine Synthese der bis dahin erhaltenen Befunde bei.

Der jeweilige Zustand hängt von der Orientierung des magnetischen Feldes $\mathrm{H}$ zur Richtung $\Delta$ des Antiferromagnetismus ab, d.h. zur bevorzugten (Faden-)Richtung des Kristallgitters, der Vorzugslage, in der die atomaren Momente bei Abwesenheit eines äußeren Feldes $H$ parallel und antiparallel orientiert sind. Wenn $\mathrm{H}$ senkrecht $\mathrm{zu} \Delta$ wirkt, erhält man die Kurve $A$ in Abbildung $6^{[7]}$. Ist $\mathrm{H}$ parallel zu $\Delta$, ergibt sich Kurve $\mathrm{B}^{[10]}$. Für einen regellos polykristallinen Stoff schließlich findet man die mittlere Kurve $C$, wobei man für die Néel-Temperatur $\Theta_{p}$ die Existenz eines scharfen Maximums der spezifischen Wärme sowie der Suszeptibilität anzunehmen hat. Unterhalb der Temperatur dieser Höchstwerte existiert der antiferromagnetische Ordnungszustand, der durch Neutronenbeugung nachgewiesen werden kann.

Heute kennt man eine sehr große Zahl antiferromagnetischer Stoffe. Im allgemeinen sind sie zusammengesetzt aus Übergangsmetallen und Sauerstoff oder Schwefel. Vom Standpunkt der Theorie aus sind sie äußerst beachtens- wert, nicht jedoch für irgendwelche technischen Anwendungen.

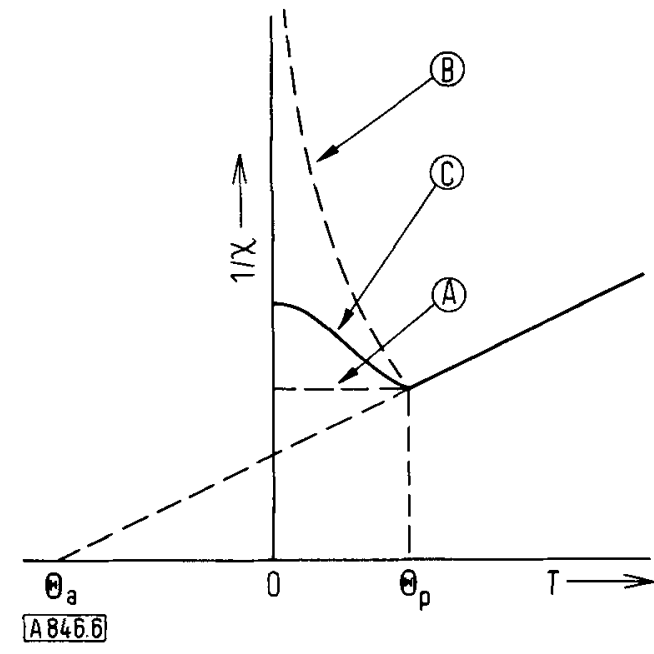

Abb. 6. Temperaturfunktion des Kehrwerts der Suszeptibilität eines antiferromagnetischen Stoffes.

\section{Andere Forschungsarbeiten auf dem Gebiet des Magnetismus}

Ich muß hervorheben, daß meine theoretischen Studien in den Jahren 1930 bis 1938 das Ziel hatten, die elementaren Wechselwirkungsenergien $w$ zwischen zwei gleichen oder verschiedenen nächstbenachbarten magnetischen Atomen $\mathrm{zu}$ bestimmen. Man durfte hoffen, auf diesem Wege ein allgemeines Gesetz zu finden, das w als Funktion des Abstandes der gekoppelten Atome wiedergibt. Augenscheinlich lag in solchem Vorgehen jedoch eine recht weitgehende Vereinfachung, denn diese Hoffnung war damals reichlich phantastisch. Trotzdem wurden positive Ergebnisse erzielt, die zu beachtenswerten Korrelationen zwischen den Anomalien der thermischen Ausdehnung, der Wirkung eines mechanischen Druckes und der Änderung der molekularen Felder mit der Temperatur fiihrten. Heute noch sind diese Zusammenhänge durchaus aktuell geblieben.

Von 1938 ab verließ ich für etwa acht Jahre das Problem der Wechselwirkungen und der lokalen Molekularfelder, um anderen Erscheinungen nachzugehen: Rayleigh-Gesetze, feinkörniges Pulver, physikalische Rolle innerer Streufelder. Ich nenne hier nur diejenigen Arbeitsrichtungen aus jener Zeit, die bemerkenswerte Ergebnisse erbracht haben.

Für den Schutz von Schiffen gegen magnetische Minen hatte ich ein Verfahren vorgeschlagen, bei dem die Schiffe permanent magnetisiert werden im gleichen Betrage, aber entgegengesetzt zu ihrer Magnetisierung durch das magnetische Erdfeld. Daraus folgte mein Interesse an den Gesetzmäßigkeiten der Magnetisierung ferromagnetischer Stoffe in schwachen Feldern, die als Rayleigh-Gesetze bekannt sind. Ich konnte dafür eine Deutung finden ${ }^{[12]}$, die auf der Fortbewegung der Bloch-Wände in einer statistisch unregelmäßig gestörten Umgebung beruht.

Auch untersuchte ich systematisch die bis dahin weitgehend vernachlässigte Rolle der inneren magnetischen 
Streufelder. Es gelang so, eine verbesserte Theorie der Gesetzmäßigkeiten für die Annäherung der ,technischen Magnetisierungskurve" an die Sättigungsmagnetisierung zu entwickeln, die der regellosen Richtungsverteilung der Kristallkörner ${ }^{[13]}$ und der Anwesenheit unmagnetischer Hohlräume oder Einschlüsse ${ }^{[14]}$ Rechnung trägt. Ferner ergab sich dabei eine allgemeinere Theorie des Koerzitivfeldes mit Berücksichtigung der unregelmäßig verteilten inneren mechanischen Spannungen sowie der unmagnetischen Hohlräume und Einschlïsse ${ }^{[15]}$. In der gleichen Gedankenrichtung schlug ich eine Theorie der Magnetisierung von ferromagnetischen Einkristallen vor ${ }^{[16]}$, wobei ich in Schritten vorging, von denen jeder gekennzeichnet war durch die Zahl der Phasen, d.h. durch die Zahl der verschiedenen Arten von spontan magnetisierten Elementarbereichen: Da sich diese Theorie mit den experimentellen Befunden vollständig deckte, setzte sie einen Schlußstrich unter eine zuvor sehr schlecht verstandene Erschejnung.

Im Jahre 1942 vermochte ich schließlich zu zeigen, daß hinreichend kleine ferromagnetische Teilchen nur einen einzigen Elementarbereich enthalten können und sich deshalb je nach ihren Abmessungen so verhalten müssen wie ein "superparamagnetischer" Stoff oder wie eine Ansammlung kleiner permanenter Magnete, die makroskopisch eine Hystereseschleife mit sehr hohem Koerzitivfeld aufweist. Von magnetisch weichen Eisenteilchen ausgehend, die lediglich eine Anisotropie der äußeren Gestalt besitzen, kann man so gute Dauermagnete herstellen. Deren Eigenschaften und Anwendungsmöglichkeiten hat $L$. Weil weiterentwickelt. Die Veröffentlichung aller dieser Arbeiten verzögerte sich bis $1947^{[17]}$, sowohl wegen der deutschen Besetzung Frankreichs als auch zur Wahrung industrieller Rechte.

\section{Magnetische Eigenschaften der ferritischen Spinelle}

Nach dem Studium eines Beitrages von Verwey und Heilmann $^{[18]}$ über die Struktur der Ferrite wendete ich mich wieder der Untersuchung dieser Stoffe zu. Die Ferrite $\mathrm{Fe}_{2} \mathrm{O}_{3} \mathrm{MO}$ mit Spinell-Struktur, wobei $\mathrm{M}$ ein zweiwertiges Metall bedeutet, gliedern sich hinsichtlich ihrer magnetischen Eigenschaften in zwei Gruppen: paramagnetische Ferrite, mit $\mathrm{M}=\mathrm{Zn}$ oder $\mathrm{Cd}$, und ferromagnetische Ferrite mit $\mathrm{M}=\mathrm{Mn}, \mathrm{Co}, \mathrm{Ni}$ usw. Diese zweite Gruppe hat große technische Bedeutung gewonnen, denn es handelt sich um ferromagnetische elektrische Isolatoren. Ein nicht geringeres theoretisches Interesse haben sie deshalb erlangt, weil ihre seltsamen Eigenschaften von denen klassischer Ferromagnetika erheblich abweichen: Ihr atomares Sättigungsmoment von 1 bis $5 \mu_{\mathrm{B}}\left(\mu_{\mathrm{B}}=\right.$ Bohr-Magneton) ist viel schwächer als das gesamte magnetische Dipolmoment der im Molekül enthaltenen Ionen, das zwischen 10 und $15 \mu_{\mathrm{B}}$ beträgt. Darüber hinaus folgt der Kehrwert der Suszeptibilität in Abhängigkeit von der Temperatur oberhalb der Curie-Temperatur einer Hyperbel, deren außergewöhnliche Krümmung von der T-Koordinate weggerichtet ist und deren Asymptote für hohe Temperaturen auf der $\mathrm{T}$ Koordinate eine negative absolute Temperatur abschneidet.
Fräulein Serres, der sehr schöne experimentelle Untersuchungen an Ferriten zu verdanken sind, erklärte das Verhalten der Funktion $(1 / \chi, T)$ durch Überlagerung eines temperaturunabhängigen Paramagnetismus der $\mathrm{Fe}^{3+}$-Ionen wie in $\alpha-\mathrm{Fe}_{2} \mathrm{O}_{3}{ }^{[19]}$. Da jedoch dieser Paramagnetismus keine atomare Eigenschaft ist, versteht man nicht, warum die $\mathrm{Fe}^{3+}$-Ionen in den Ferriten den gleichen konstanten Paramagnetismus beibehalten wie im $\alpha-\mathrm{Fe}_{2} \mathrm{O}_{3}$.

Hinsichtlich der Kristallstruktur sei erwähnt, daß die Metall-Ionen in einer dichtesten kubischen Packung von Sauerstoff-Ionen Zwischengitterplätze $\mathrm{A}$ mit vier nächsten $\mathrm{O}^{2-}$ und $\mathrm{B}$ mit sechs nächsten $\mathrm{O}^{2-}$ besetzen. Es gibt nun zwei Arten von Ferriten: In den normalen Ferriten besetzen die beiden $\mathrm{Fe}^{3+}$-Ionen des Moleküls die beiden Plätze $\mathrm{B}$ und das Ion $\mathrm{M}^{2+}$ den Platz $\mathrm{A}$; in den inversen Ferriten besetzt eines der $\mathrm{Fe}^{3+}$-Ionen den Platz $\mathrm{A}$, das andere einen der Plätze B. Durch Röntgen-Analyse sind Verwey und Heilmann zu dem Schluß gekommen, daß die ferromagnetischen Ferrite invers, die paramagnetischen normal strukturiert sind.

\section{Grundlagen der Theorie des Ferrimagnetismus}

Zur Deutung der Eigenschaften ferrimagnetischer Stoffe ging ich von der Annahme aus ${ }^{[20]}$, daß die vorherrschenden magnetischen Wechselwirkungen zwischen den Ionen auf den Gitterplätzen A und den Ionen auf den Gitterplätzen $B$ wirksam und im wesentlichen negativ sind. Bei der Temperatur $0^{\circ} \mathrm{K}$ zwingen starke Wechselwirkungskräfte die Momente der A-Ionen zur parallelen Ausrichtung unter sich mit einer Resultierenden $\mathrm{M}_{\mathrm{as}}$, antiparallel zur gleichartigen Resultierenden $\mathrm{M}_{\mathrm{bs}}$ der Momente der BIonen. Die meßbare spontane Magnetisierung ist dann die Differenz $\left|\mathrm{M}_{\mathrm{as}}-\mathrm{M}_{\mathrm{bs}}\right|$.

$\mathrm{Zu}$ bedeutsamen Folgerungen aus diesen Hypothesen kam ich durch Anwendung des Begriffs der lokalen Molekular-
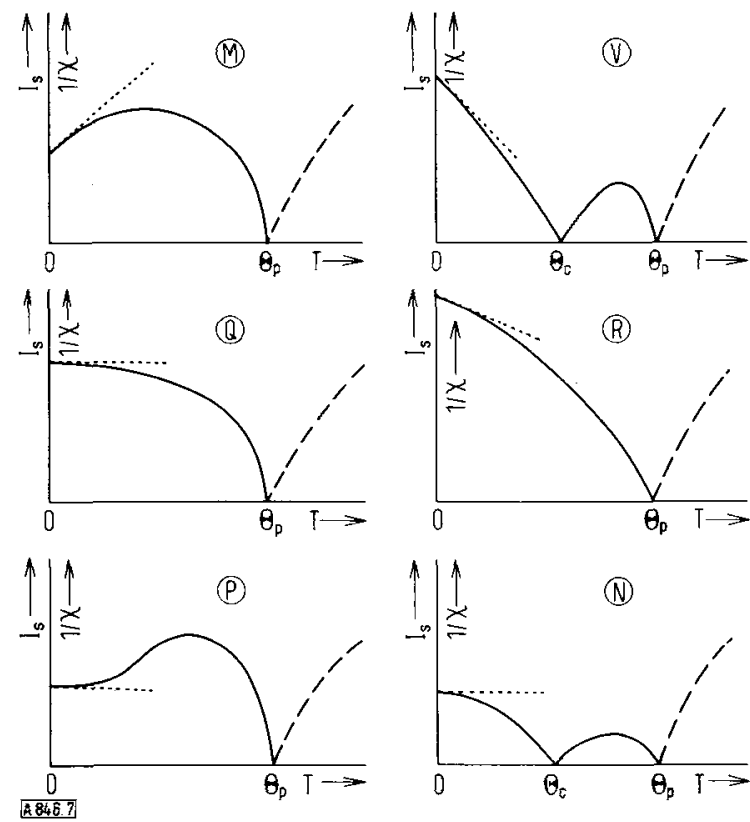

Abb. 7. Temperaturfunktion der spontanen Magnetisierung und des Kehrwerts der Suszeptibilität eines ferrimagnetischen Stoffes: Mögliche verschiedene Typen (vgl. Abb. 8). 


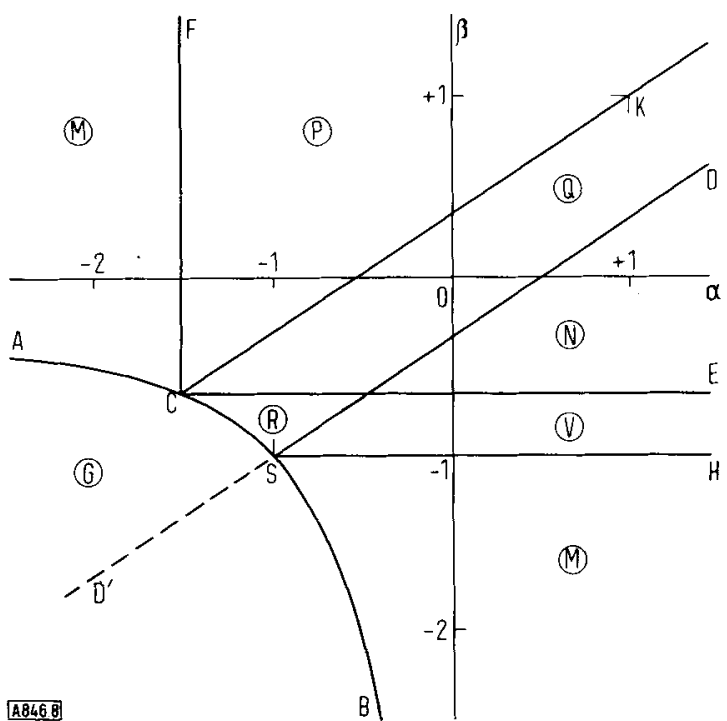

Abb. 8. Bereiche $\alpha$ und $\beta$ in der $(\alpha, \beta)$-Ebene für die Kurventypen von Abb. 7.

felder, den ich früher ${ }^{[2]}$ entwickelt hatte. Hierfür werden mit $\lambda$ und $\mu(\lambda+\mu=1)$ die relativen Anteilzahlen der als identisch angenommenen, magnetischen Ionen in den Teilgittern $\mathrm{A}$ und $\mathrm{B}$ bezeichnet, mit $\mathrm{n}, \mathrm{n} \alpha$ und $\mathrm{n} \beta$ die Koeffizienten des Molekularfeldes, die den Wechselwirkungen $\mathrm{AB}$, $\mathrm{AA}$ und $\mathrm{BB}$ entsprechen. Es zeigte sich, daß die spontane Magnetisierung als Funktion der Temperatur in starker Schematisierung die in Abbildung 7 angedeuteten Formen aufweisen müßte, wenn dort die eingekreisten Symbole jeweils dem entsprechenden Bereich der $(\alpha, \beta)$-Ebene der Abbildung 8 zugeordnet werden und der Bereich (G) dabei einem Paramagnetismus entspricht. Alle Abschnitte der Kurven in Abbildung 7 wurden später auch experimentell aufgefunden.

Oberhalb der Curie-Temperatur $\Theta_{p}$ werden die Ferrite paramagnetisch mit einer Suszeptibilität $\chi$ gemäß

$\frac{1}{\chi}=\frac{T}{C}+\frac{1}{\chi_{0}}-\frac{\sigma}{T-\Theta}$

Darin bedeuten

$\frac{1}{\chi_{0}}=n\left(2 \lambda \mu-\lambda^{2} \alpha-\mu^{2} \beta\right)$

$\sigma=\mathrm{nC}^{2} \lambda \mu[\lambda(1+\alpha)-\mu(1+\beta)]^{2}$

$\Theta=n C \lambda \mu(2+\alpha+\beta)$

Wir sehen hier also Stoffe vor uns, deren Ferromagnetismus in sehr beachtenswerter Weise auf negativen Wechselwirkungen beruht und die sich daher von den klassischen ferromagnetischen Stoffen grundsätzlich unterscheiden, so $\mathrm{da} ß$ eine besondere Namensgebung gerechtfertigt erschien. Ich schlug die Bezeichnung "ferrimagnetische Stoffe" vor.

\section{Vergleich der theoretischen mit den experimentellen Befunden}

Diese Theorie machte es möglich ${ }^{[20]}$, die Eigenschaften der Ferrite von Magnesium, Blei und Calcium sowie die des Magnetits $\mathrm{Fe}_{3} \mathrm{O}_{4}$ und des Mangan-Antimonits $\mathrm{Mn}_{2} \mathrm{Sb}$ un-

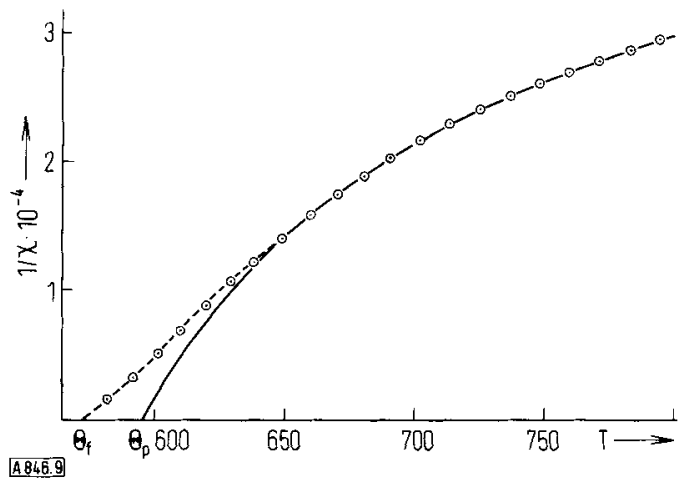

Abb. 9. Temperaturfunktion des Kehrwerts der Suszeptibilität des Magnetits (angepaßte Kurve und Meßwerte). Man beachte den Unterschied zwischen der ferromagnetischen und der paramagnetischen Curie-Temperatur!

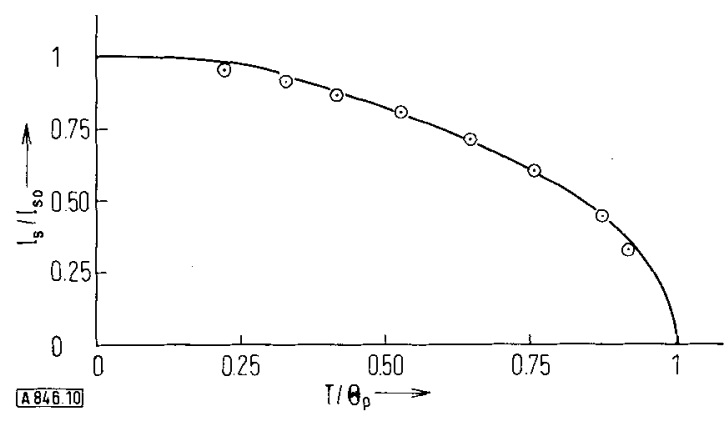

Abb. 10. Temperaturfunktion der spontanen Magnetisierung des Magnetits (Meßwerte und Kurve, die mit den Koeffizienten des Molekularfeldes berechnet wurde, welche aus den gemessenen Suszeptibilitäten abgeleitet sind).

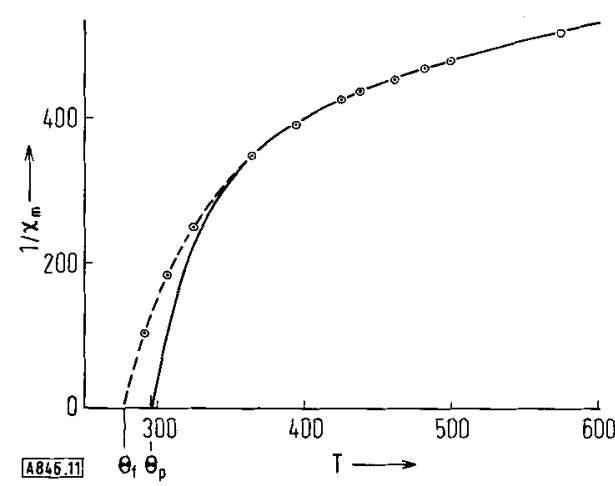

Abb. 11. Temperaturfunktion des Kehrwerts der Suszeptibilität des Mangan-Antimonits $\mathrm{Mn}_{2} \mathrm{Sb}$ (Meßwerte und angepaßte Kurve).

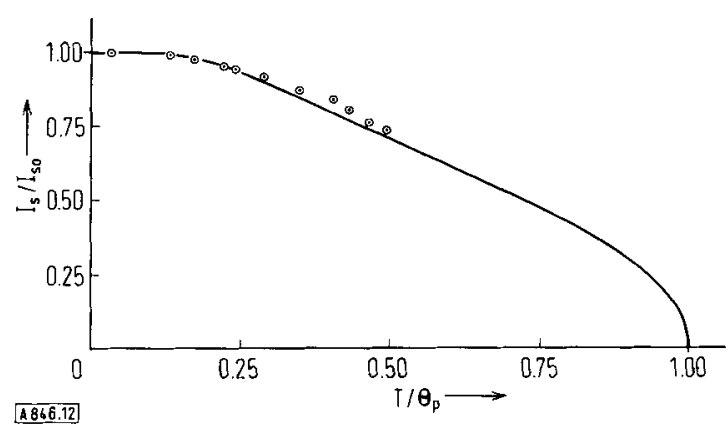

Abb. 12. Temperaturfunktion der spontanen Magnetisierung des Mangan-Antimonits $\mathrm{Mn}_{2} \mathrm{Sb}$ (Meßwerte und Kurve, die mit den Koeffizienten des Molekularfeldes berechnet wurde, welche aus den gemessenen Suszeptibilitäten abgeleitet sind). 
mittelbar zu erklären. Die Abbildungen 9, 10, 11 und 12 zeigen als Beispiele den Vergleich der experimentellen mit den berechneten Werten im Falle von $\mathrm{Fe}_{3} \mathrm{O}_{4}$ und $\mathrm{Mn}_{2} \mathrm{Sb}$ für die Temperaturabhängigkeit der spontanen Magnetisierung und des Kehrwerts der Suszeptibilität. In beiden Fällen wurden die gleichen Koeffizienten $n, \alpha$, $\beta$ verwendet. Sehr ermutigende erste Ergebnisse veranlaßten ausgedehnte experimentelle Bemühungen zur Prüfung der Theorie, da die damals verfügbaren Daten noch recht spärlich waren.

In den rein inversen Ferriten $\mathrm{Fe}_{2} \mathrm{O}_{3} \mathrm{MO}$ mit $\mathrm{M}=\mathrm{Mn}, \mathrm{Fe}$, $\mathrm{Co}, \mathrm{Ni}$ muß die Sättigungsmagnetisierung bei der Temperatur $0^{\circ} \mathrm{K}$ einfach durch das magnetische Moment des Ions $\mathrm{M}^{2+}$ gegeben sein, da ja die Momente der beiden $\mathrm{Fe}^{3+}$ Ionen, die zwei verschiedene Gitterplätze besetzen, sich gegenseitig kompensieren. Abbildung 13 gibt den Vergleich von Theorie und Experiment wieder. Die dort eingetragenen Punkte sind Meßwerte ${ }^{[21]}$. Die Gerade entspricht den theoretischen Voraussagen unter Annahme eines allein wirksamen Spinmoments, der schraffierte Bereich den verfeinerten theoretischen Voraussagen, bei denen auch ein nicht voll blockiertes Bahnmoment rechnerisch berücksichtigt ist, das aus dem effektiven Moment der paramagnetischen Salze abgeleitet wurde. Die Übereinstimmung ist sehr befriedigend. Für Kupfer-Ferrit zeigt die Erfahrung, daß die Sättigungsmagnetisierung von der thermischen Vorbehandlung abhängt: Bei langsamer Abkühlung entsteht die inverse Struktur; bei hoher Temperatur verteilen sich jedoch die $\mathrm{Fe}^{3+}$-Ionen unregelmäßig auf Plätze $\mathrm{A}$ und $B$, da der Energieunterschied zwischen der normalen und der inversen Struktur nur in der Größenordnung kT liegt. Eine genaue Analyse dieser Erscheinung hat diese Deutung bestätigt, also die Theorie des Ferrimagnetismus zusätzlich gestützt ${ }^{[22]}$.

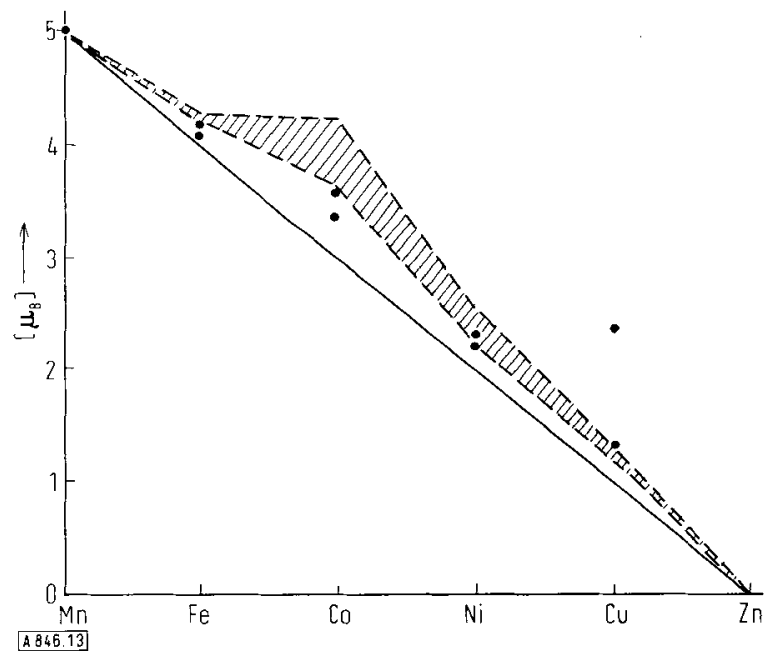

Abb. 13. Absolute Sättigungsmagnetisierung einiger Ferrite mit verschiedenen Ionen $\mathrm{M}^{2+}$.

Außerdem ist es gelungen, für diese Ferrite den gemessenen Temperaturverlauf der spontanen Magnetisierung ${ }^{[23]}$ sowie der Suszeptibilität oberhalb der Curie-Temperatur ${ }^{[24]}$ zwanglos zu interpretieren. Die Abbildungen 14 und 15 bestätigen die gute Übereinstimmung zwischen Theorie und Experiment für die drei Ferrite des Eisens, des Kobalts und des Nickels.

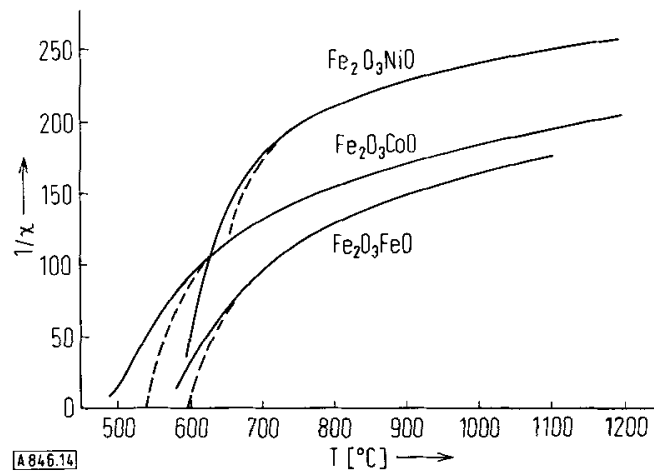

Abb. 14. Temperaturfunktion des Kehrwerts der Suszeptibilität einiger Ferrite (MeBwerte: ausgezogene Kurve, Rechenwerte: gestrichelte Kurve).

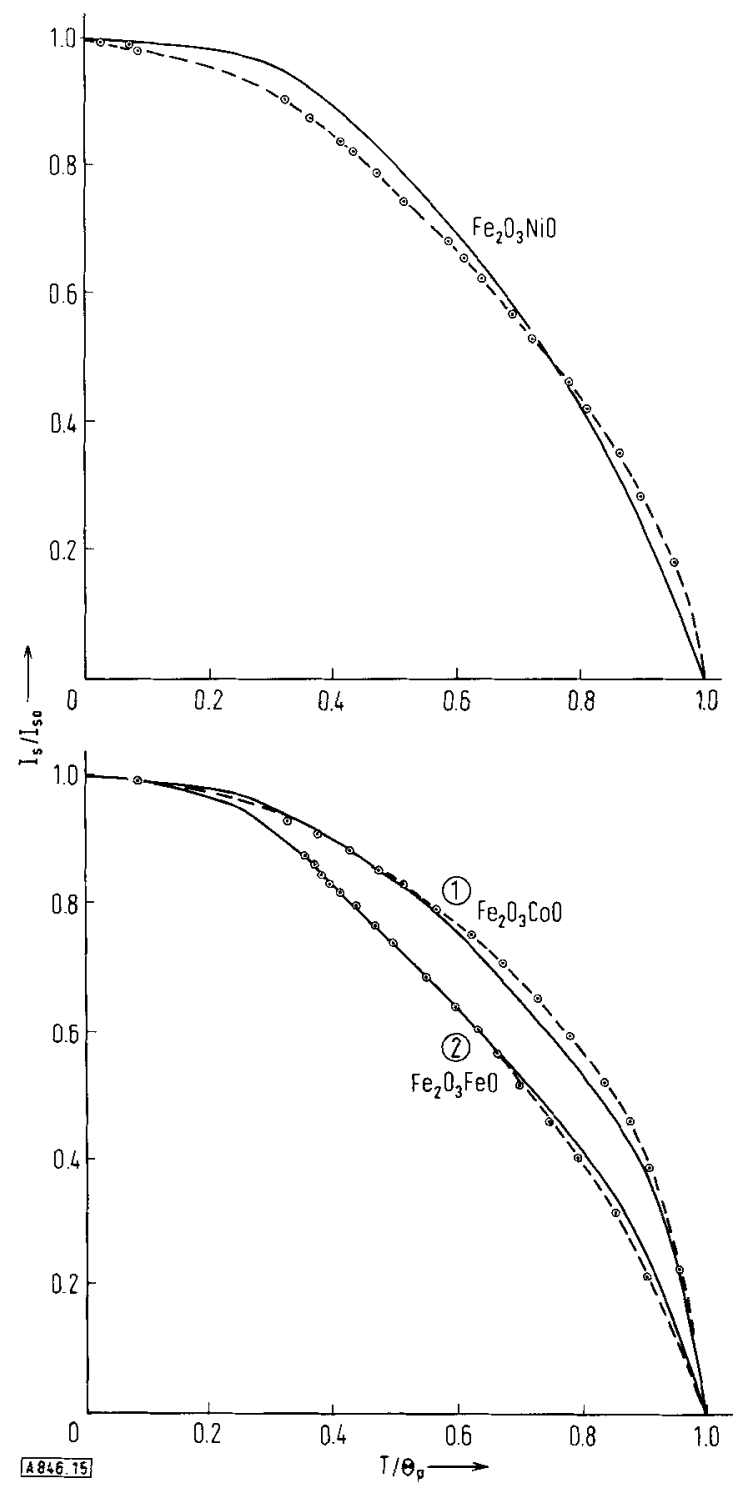

Abb. 15. Temperaturfunktion der spontanen Magnetisierung einiger Ferrite (Meßwerte und Kurven, die mit den Koeffizienten des Molekularfeldes berechnet wurden, welche aus den gemessenen Suszeptibilitäten abgeleitet sind).

Shull und Mitarbeiter ${ }^{[25]}$ wiesen durch Neutronenbeugung ergänzend auch nach, daß die magnetischen Atommomente an den Plätzen A tatsächlich - wie erwartet - antiparallel gerichtet sind zu den Atommomenten an den Plätzen B. 
Ferner ermöglichte die Theorie des Ferrimagnetismus, auch das Verhalten der Misch-Ferrite zu erklären, u. a. des Nickel-Zink-Ferrits $\left[\mathrm{Fe}_{2},(1-\mathrm{x}) \mathrm{Ni}, \mathrm{xZn}, \mathrm{O}_{4}\right]$. Ein ZinkIon, das ein Nickel-Ion ersetzt, nimmt dort einen Platz A ein, da dies der vom Zink bevorzugte Platz ist. Gleichzeitig begibt sich das $\mathrm{Fe}^{3+}$-Ion vom Platz $\mathrm{A}$ zum Platz $\mathrm{B}$, der vom Nickel freigemacht wurde, wobei sich zugleich sein Moment $5 \mu_{\mathrm{B}}$ um $180^{\circ}$ dreht. Die resultierende Änderung des Sättigungsmoments erreicht demnach $8 \mu_{\mathrm{B}}$ als Differenz zwischen $10 \mu_{\mathrm{B}}$ infolge der Umkehrung von $5 \mu_{\mathrm{B}}$ und $2 \mu_{\mathrm{B}}$ infolge des Verlustes des $\mathrm{Ni}^{2+}$-Ions. Die Neigung der Tangente für kleine $x$-Werte an der Meßkurve der Sältigungsmomente als Funktion von $x$ beträgt daher in diesem Fall $8 \mu_{\mathbf{B}}$. Das Experiment bestätigt diese Voraussage $^{[26]}$ (Abb. 16).

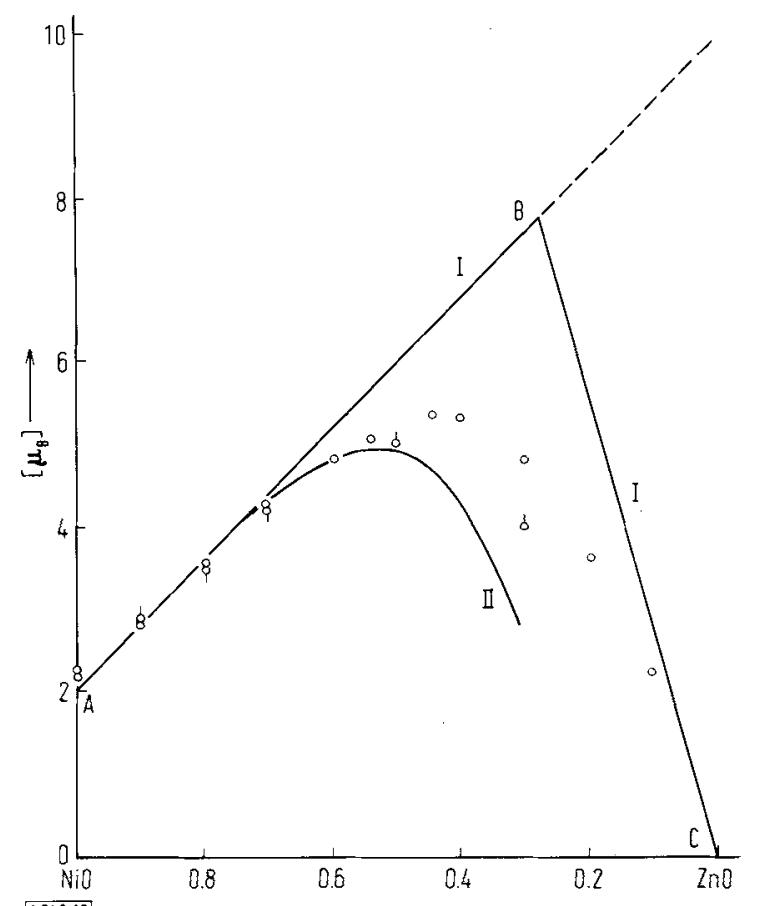

Abb. 16. Sättigungsmagnetisierung einiger Nickel-Zink-Ferrite als Funktion des Zn-Gehalts (Meßwerte und berechnete Kurven).

\section{Der Fall des Pyrrhotins}

Aufgrund derselben Theorie konnte bald darauf das Rätsel des Pyrrhotins (Magnetkies) $\mathrm{Fe}_{7} \mathrm{~S}_{8}$ gelöst werden, einer ferromagnetischen Verbindung, die $P$. Weiss ${ }^{[27]}$ schon lange vorher untersucht hatte. Sie besitzt ein schwaches Sättigungsmoment von ungefähr $3 \mu_{\mathrm{B}}$, während man nach der chemischen Formel einen fast zehnfach größeren Betrag erwartete. Der Pyrrhotin hat die gleiche Kristallstruktur des Typs B 8 nach Ewald wie das Sulfid FeS, das antiferromagnetisch ist. In diesem Sulfid sind die senkrecht zur ternären Achse aufeinander folgenden Ebenen mit Fe-Atomen alternierend antiparallel magnetisiert. F. Bertaut ${ }^{[28]}$ konnte jedoch zeigen, daß Pyrrhotin in Wirklichkeit eine Verbindung mit Lücken im Gitter ist, die richtiger mit der Formel $\mathrm{Fe}_{7} \mathrm{~S}_{8} \mathrm{~T}$ beschrieben wird, worin $\mathrm{T}$ eine solche Lücke bedeutet, d. h. einen Gitterplatz, der in der Verbindung FeS durch ein $\mathrm{Fe}^{2+}$-Ion besetzt wäre, in $\mathrm{Fe}_{7} \mathrm{~S}_{8}$ aber frei bleibt. Bei tiefer Temperatur ordnen sich die Lïcken im Kristall- gitter regelmäßig an, so wie es in Abbildung 17 für die geradzahligen Fe-Ebenen dargestellt ist: Die Lücken sind bestrebt, sich dabei möglichst weit voneinander zu entfernen. Auf den ungeradzahligen Eisenebenen sind hingegen alle Plätze besetzt. Die beiden, im Falle von FeS äquivalenten Teilgitter sind hier also verschieden, so daß man bei $\mathrm{Fe}_{7} \mathrm{~S}_{8}$ einen echten Ferrimagnetismus vor sich hat. Wenn die Temperatur auf über $900^{\circ} \mathrm{K}$ steigt, verschwindet dieser Ordnungszustand des Kristallgitters und der Lücken: Die beiden Teilgitter werden statistisch äquivalent. Entsprechend rückt die paramagnetische Susżeptibilität des Pyrrhotins nahe heran an die von FeS.

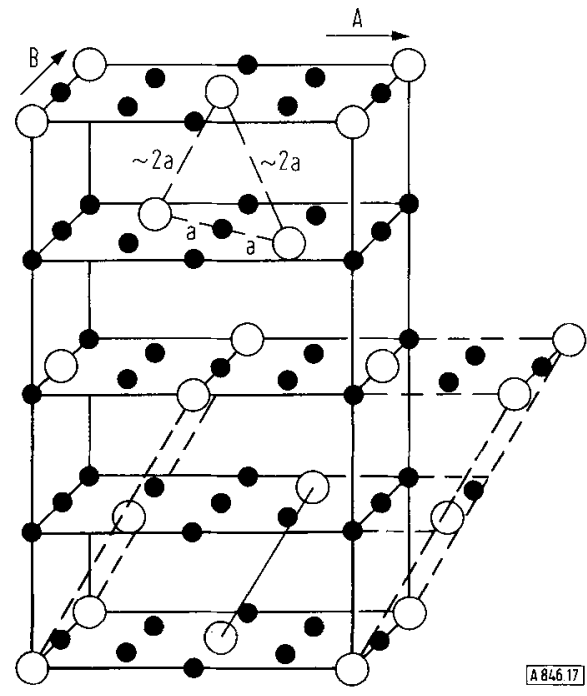

Abb. 17. Kristallstruktur des Pyrrhotins bei tiefer Temperatur. Die vollen schwarzen Kreise stellen die Eisenatome dar, die offenen Kreise die Gitterlïcken. Die Fe-Ebenen geradzahliger Ordnung und die Sauerstoffatome sind nicht wiedergegeben.

\section{Entdeckung der ferritischen Granate}

Das schönste Beispiel für Ferrimagnetismus haben zweifellos die ferritischen Granate geliefert. Diese Stoffe der allgemeinen Formel $\mathrm{Fe}_{5} \mathrm{M}_{3} \mathrm{O}_{12}$, wobei $\mathrm{M}$ ein dreiwertiges Ion eines Seltenerdmetalls bedeutet, bilden eine wichtige Gruppe magnetischer Verbindungen, deren Eigenschaften auf einen Ferrimagnetismus von drei Teilgittern zurückgeführt werden können. Da sich ferner die Metallionen in den Granaten mit vielfacher Variationsmöglichkeit durch andere Ionen ersetzen lassen, kommt diesen Verbindungen auch für das theoretische Studium der Wechselwirkungen große Bedeutung zu. Unter dem Aspekt der technischen Anwendungen muß hervorgehoben werden, da $B$ sie ausgezeichnete Isolatoren sind, in großen Kristallen gezüchtet werden können und sehr scharfe Resonanzlinien aufweisen; sie werden deshalb in verschiedenartigen Hochfrequenzgeräten verwendet.

Die Geschichte ihrer Entdeckung beginnt im Jahre 1950 in Straßburg, wo Forestier und Guiot-Guillain ${ }^{[29]}$ durch Erhitzen einer äquimolaren Mischung von $\mathrm{Fe}_{2} \mathrm{O}_{3}$ und $\mathrm{M}_{2} \mathrm{O}_{3}(\mathrm{M}=$ Seltenerdmetall) stark ferromagnetische Stoffe erhielten mit Curie-Temperaturen von 520 bis $740^{\circ} \mathrm{K}$. Die genannten Forscher vermuteten eine Kristallstruktur vom Typ des Perovskit ${ }^{[30]}$ und kamen zu einem überraschenden 
Befund $^{[31]}$ : Mit $\mathrm{M}=\mathrm{Yb}, \mathrm{Tm}, \mathrm{Dy}, \mathrm{Gd}$ oder Sm erhielten diese Stoffe zwei Curie-Temperaturen im Abstand von etwa $100^{\circ}$ mit regelmäßiger Abhängigkeit vom Atomradius von $\mathrm{M}$.

Diese Beobachtungen zogen die Aufmerksamkeit der Forscher in Grenoble auf sich. Pauthenet und Blum ${ }^{[32]}$ stellten einen Gadolinium-Ferrit her und wiesen nach, da $\beta$ diese Verbindung außer den zwei Curie-Temperaturen $\Theta_{1}$ $=570^{\circ} \mathrm{K}$ und $\Theta_{2}=678^{\circ} \mathrm{K}$ eine dritte Umwandlungstemperatur $\Theta_{3}=306^{\circ} \mathrm{K}$ besitzt, die man im Sinne des Ferrimagnetismus als eine Kompensationstemperatur aufzufassen hat, d.h. als eine Temperatur, bei der die spontane Magnetisierung ihr Vorzeichen wechselt (Typen (V) und (Q) in Abb. 7).

Zur Deutung der Befunde nahm ich an ${ }^{[33]}$, daß die $\mathrm{Fe}^{3+}$ Ionen ein von $\mathrm{M}$ unabhängiges ferrimagnetisches Teilgitter A bilden, dessen resultierende spontane Magnetisierung

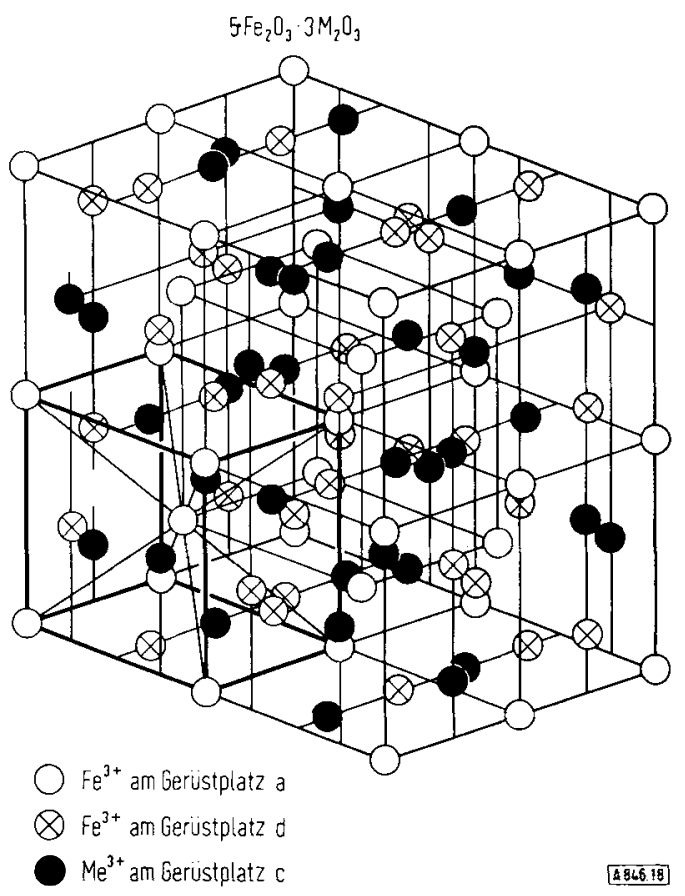

Abb. 18. Kristallstruktur ferritischer Granate.

antiparallel zum Teilgitter $\mathrm{B}$ der $\mathrm{M}^{3+}$-Ionen gerichtet ist. Das molekulare Feld $h_{A}$, das die orientierende Wirkung von $A$ auf $B$ ausübt, muß dabei hinreichend schwach sein, so daß mindestens oberhalb $100^{\circ} \mathrm{K}$ die Magnetisierung von $B$ nahezu gleich $C_{B} h_{A} / T$ wird, und die Temperatur $\Theta_{3}$ diejenige ist, wo die Magnetisierung von $B$ gleich und antiparallel zur Magnetisierung von A wird. Daraus folgte insbesondere, da $\beta$ die Temperatur $\Theta_{3}$ um so tiefer zu erwarten war, je kleiner die Curie-Konstante $\mathrm{C}_{\mathrm{B}}$ der $\mathrm{M}^{3+}$-Ionen ist. In Übereinstimmung mit diesen Voraussagen ergab sich einige Tage später, daß auch die Ferrite des Dysprosiums und des Erbiums Kompensationstemperaturen bei 246 bzw. $70^{\circ} \mathrm{K}$ aufweisen ${ }^{[34]}$. Neben diesem Erfolg hatte sich jedoch gezeigt, daß die hier wirksame Kristallstruktur keineswegs mit derjenigen des Perovskits $\mathrm{FeMO}_{3}$ identisch sein konnte.

Mit einiger Wahrscheinlichkeit mußte es sich um eine andere Verbindung in Mischung mit Perovskit handeln. Von diesem Gedanken ausgehend, konnten Bertaut und Forrat $^{[35]}$ im Januar 1956 nachweisen, daß eine kubische Verbindung $\mathrm{Fe}_{5} \mathrm{M}_{3} \mathrm{O}_{12}$ der Raumgruppe $\mathrm{O}_{\mathrm{h}}^{10}$ (Ia 3d) mit acht Molekülen in der Elementarzelle vorliegt, deren Struktur identisch ist mit der Struktur der Edelsteine, die als Granate bekannt sind (Abb. 18). Die primäre ferrimagnetische Anordnung besteht aus $24 \mathrm{Fe}^{3+}$-Ionen auf den Plätzen d, umgeben von $4 \mathrm{O}^{2-}$-Ionen und $16 \mathrm{Fe}^{3+}$-Ionen auf den Plätzen a, umgeben von $6 \mathrm{O}^{2-}$-Ionen. Diese durch starke Kopplungskräfte bedingte Ordnung hat magnetische Eigenschaften, die weitgehend unabhängig sind von der Natur der Ionen $\mathrm{M}^{3+}$, mit denen sie nur durch schwache Kopplungskräfte verbunden ist. Man kann dieses Modell magnetisch bestätigen, indem man als $M$ ein unmagnetisches Ion wie Y oder Lu einbaut.

\section{Deutung durch einen Ferrimagnetismus bei drei Teilgittern}

Die Verallgemeinerung der Theorie des Ferrimagnetismus für drei Teilgitter und die Darstellung der Wechselwirkungen mit neun lokalen Molekularfeldern, von denen sechs unabhängig sind, bereiten keine Schwierigkeiten. Die so berechneten Kurven decken sich sehr befriedigend mit den experimentell gefundenen. Für den Temperaturgang der spontanen Magnetisierung einiger Granate zeigt das Abbildung 19, die der grundlegenden Veröffentlichung von

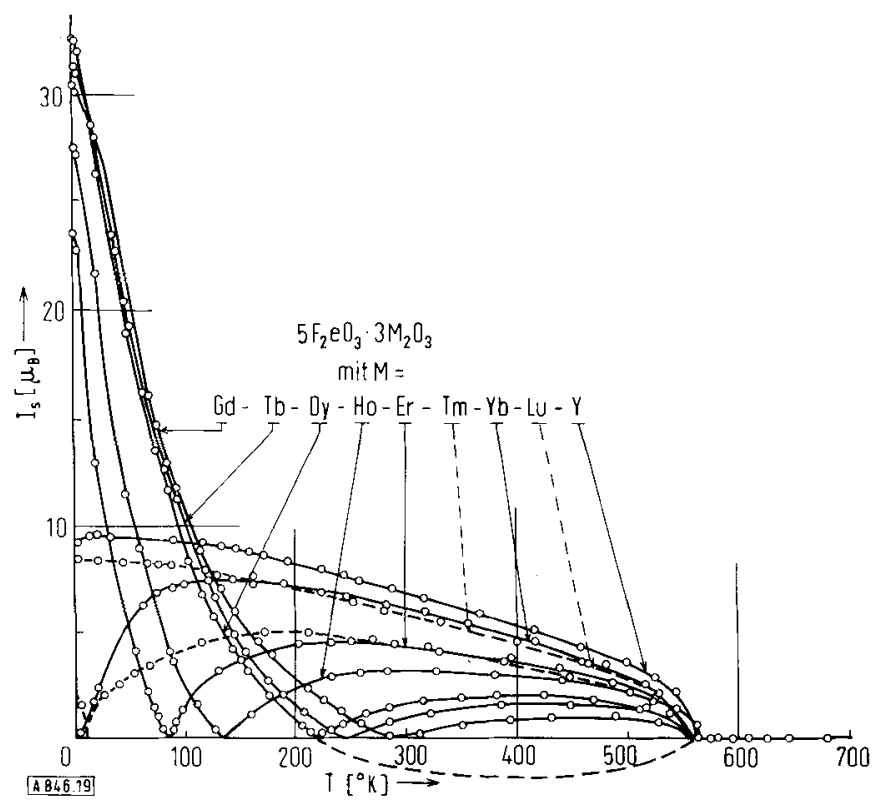

Abb. 19. Temperaturfunktion der spontanen Magnetisierung einer Reihe ferritischer Granate. Man beachte den Gang der Kompensationstemperatur mit der Ordnungszahl des Seltenerdmetalls!

Pauthenet ${ }^{[36]}$ entnommen ist. Die weitgehende Übereinstimmung der Curie-Temperaturen entspricht sehr gut der soeben erläuterten Auffassung, daß die Natur der $\mathrm{M}^{3+}$. Ionen die ferrimagnetische Anordnung der $\mathrm{Fe}^{3+}$-Ionen nicht merklich beeinflußt. Im paramagnetischen Bereich oberhalb der Curie-Temperatur decken sich die berechneten mit den experimentell ermittelten Kurven ebenso befriedigend (Abb. 20). 


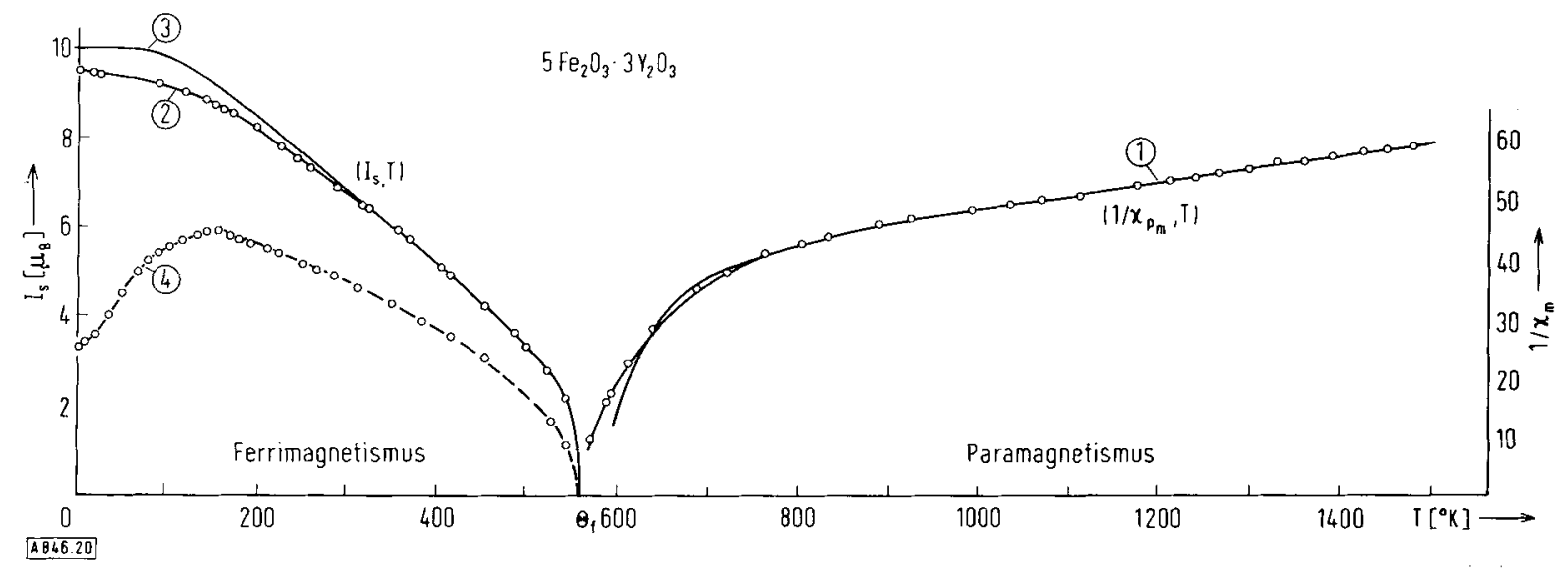

Abb. 20. Yttrium-Granat. Temperaturfunktion der spontanen Magnetisierung und des Kehrwerts der Suszeptibilität; Meßwerte und Kurven, die mit den gleichen Koeffizienten des Molekularfeldes berechnet wurden. Kurve 4 bezieht sich auf Yttrium-Granat mit einem geringen Gehalt an Gadolinium.

\section{Der Super-Austausch}

Sowohl für den Antiferromagnetismus als auch für den Ferrimagnetismus gestattet die Theorie, aus experimentellen Kurven die elementare Wechselwirkungsenergie w für zwei nächstbenachbarte Atome zu entnehmen. Die entsprechende Rechnung wurde für ferritische Spinelle und einige antiferromagnetische Stoffe wie $\mathrm{MnO}$ oder NiO ausgeführt. Sie brachte neue Erkenntnisse ${ }^{[37]}$ : In diesen Stoffen sind die Wechselwirkungen zwischen benachbarten Eisen-Ionen negativ. Bei Ionenabständen, wo sie deutlich positiv auftreten, handelt es sich um reine Metalle oder um Legierungen. Andererseits stellte man auch die Existenz von bedeutenden Wechselwirkungen zwischen magnetischen Atomen fest, zwischen denen sich Sauerstoffatome befinden, die abschirmend wirken müßten. Die Erklärung war geradezu revolutionär: Es liegt die Wirkung des sogenannten „Super-Austauschs“ (Superexchange) vor, für die das Sauerstoffatom zwischen zwei Eisenatomen eine wesentliche Rolle spielt. Kramers ${ }^{[38]}$ hatte die Möglichkeit derartiger Wechselwirkungen schon lange vorausgesagt; nunmehr konnte ihre Existenz erstmalig klar nachgewiesen werden.

In Oxiden und Ferriten gibt es nebeneinander Austauschwechselwirkungen $\mathrm{M}-\mathrm{M}$ vom klassischen Typ und SuperAustausch vom Typ $\mathrm{M}-\mathrm{O}-\mathrm{M}$. Solche Stoffe eignen sich also vorzüglich dafür, die Theorien der verschiedenen Wechselwirkungen zu prüfen.

\section{Schlußfolgerungen}

Trotz ihrer etwas naiven Einfachheit erbrachte die Methode des lokalen Molekularfeldes unleugbare Erfolge, indem in einer gedanklich befriedigenden Weise eine große Zahl bekannter Erscheinungen miteinander verknüpft werden konnte und diese Methode auch zur Entdeckung neuer Tatsachen führte. Ich muß jedoch unterstreichen, daß alle hier erwähnten Strukturen kolineare Strukturen sind: Alle Atommagnete wirken im zeitlichen Mittel parallel oder antiparallel in der gleichen Fadenrichtung. Die Methode des lokalen Molekularfeldes läßt sich aber auch auf nichtkolineare Strukturen anwenden, z. B. diejenige des „Schrau-
ben-Magnetismus", den Yoshimori und Villain unabhängig voneinander völlig unerwartet entdeckten: Man kann auch diese Erscheinungen bemerkenswert einfach und überzeugend erklären.

Es erscheint jedoch wenig empfehlenswert, die Methode lokaler Molekularfelder auch auf sehr komplizierte Strukturen anzuwenden, z. B. auf die "Regenschirm-Struktur“, für die eine Zerlegung des Grundgitters in eine große Zahl von Teilgittern erforderlichlich wäre. Unter solchen Umständen hätte ein Atom, das einem Teilgitter angehört, in jedem der anderen Teilgitter nur sehr wenige Nachbarn, oft nur einen oder zwei. Die Methode des Molekularfeldes besteht aber wesentlich darin, die momentane Wirkung eines Atoms zu ersetzen durch die Wirkung eines „durchschnittlichen Atoms.“ Sie hat um so mehr Aussicht, zu einem richtigen Ergebnis zu führen, je größer die Zahl der unmittelbar gekoppelten Atome ist. Wahrscheinlich ist sie auch um so besser brauchbar, je größer die maßgebenden atomaren Spinmomente sind. Darüber hinaus verliert die Methode des lokalen Molekularfeldes bei Anwendung auf eine große Zahl von Teilgittern weitgehend ihren wichtigsten Vorzug: die Einfachheit.

Die Methode enthält auch tückische Fallen. Sie kann bei durchaus sinnvoller Wahl der Parameter zur Berechnung von Kurven - Temperaturgängen der spontanen Magnetisierung oder der paramagnetischen Suszeptibilität - führen, die mit den Meßdaten ausgezeichnet übereinstimmen, z. B. bis auf einige Tausendstel. Man könnte dann erwarten, daß die mit diesen Parametern abgeleiteten elementaren Wechselwirkungsenergien mit der gleichen Genauigkeit den wirklichen Beträgen in der Natur gleichkommen. Doch dabei geht man oft fehl : Man kann sich um zehn bis zwanzig Prozent oder auch um mehr irren. Vorsicht ist also geboten!

Andererseits scheint das lokale Molekularfeld als Hilfsmittel unentbehrlich zu sein, denn strenge Verfahren scheitern an unüberwindbaren Schwierigkeiten. Selbst für den einfachsten Fall ist noch keine exakte Lösung bekannt, für ein einfaches kubisches Gitter mit dem Spin 1/2 der identischen Atome und mit Beschränkung auf die Wechselwirkung zwischen den nächsten Nachbarn. Wieviel mehr gelten die Schwierigkeiten für die Granate mit 160 Atomen in der Elementarzelle, Spinbeträgen bis $5 / 2$ und mindestens sechs 
verschiedenen Kopplungskonstanten! Man sollte daher gegenüber den Methoden des lokalen Molekularfeldes einige Duldsamkeit üben, in Anbetracht der einfachen Wege, auf denen sie die Erfolge ermöglichten, denen ich die ersten Sätze dieser Schlußfolgerungen gewidmet habe.

Eingegangen am 15. Februar 1971 [A 846] Übersetzt von Prof. Dr.-Ing. M. Kersten, Braunschweig

[1] P. Weiss, C. R. Acad. Sci. Paris 143, 1137 (1906); J. Phys. (Paris) 6, $666(1907)$.

[2] L. Neel, Ann. Phys. (Paris) 17, 5 (1932).

[3] L. Néel, C. R. Acad. Sci. Paris 198,1311 (1934).

[4] L. Néel, J. Phys. (Paris) 3, 160 (1932).

[5] L. Néel, Ann. Phys. (Paris) 5, 232 (1936)

[6] N. J. Poulis, J. van den Haendel, J. Ubbink, J. A. Paulis u. C. J Gorter, Phys. Rev. 82, 52 (1951).

[7] L. Néel, C. R. Acad. Sci. Paris 203, 304 (1936).

[8] C. Squire, H. Bizette u. B. Tsai, C. R. Acad. Sci. Paris 207, 449 (1938).

[9] C. G. Shull u. S. J. Smart, Phys. Rev. 76, 1256 (1949).

[10] F. Bitter, Phys. Rev. 54, 79 (1938).

[11] J. H. van Vleck, J. Chem. Phys. 9, 85 (1941).

[12] L. Néel, Cahiers Phys. 1942, Nr. 12, 1; 1943, Nr. 13, 18.

[13] L. Néel, C. R. Acad. Sci. Paris 220,814 (1945).

[14] L. Néel, C. R. Acad. Sci. Paris 220, 738 (1945).

[15] L. Néel, Cahiers Phys. 1944, Nr. 25, 21; C. R. Acad. Sci. Paris 223 , 198 (1946); Ann. Univ. Grenoble 22, 299 (1946).

[16] L. Neel, J. Phys. (Paris) 5, 241, 265 (1944).
[17] L. Néel, C. R. Acad. Sci. Paris 224, 1488, 1550 (1947); 225, 109 (1947).

[18] E. J. W. Verwey u. E. L. Heilmann, J. Chem. Phys. 15, 174 (1947).

[19] A. Serres, Ann. Phys. (Paris) 17, 53 (1932).

[20] L. Néel, Ann. Phys. (Paris) 3, 137 (1948).

[21] P. Weiss u. R. Forrer, Ann. Phys. (Paris) 12, 279 (1929); E. W. Gorter, C. R. Acad. Sci. Paris 230, 192 (1950); C. Guillard, ibid. 229, 1133 (1949).

[22] L. Néel, C. R. Acad. Sci. Paris 230, 190 (1950).

[23] R. Pauthenet u. L. Bochirol, J. Phys. (Paris) 12, 249 (1951).

[24] M. Fallot u. P. Maroni, J. Phys. (Paris) 12, 256 (1951).

[25] C. G. Shull, E. O. Wollan u. W. A. Strauser, Phys. Rev. 81, 483 (1951).

[26] L. Néel, C. R. Acad. Sci. Paris 230, 375 (1950).

[27] P. Weiss, J. Phys. (Paris) 8, 542 (1899); 4, 469, 829 (1905); P. Weiss, u. R. Forrer, Ann. Phys. (Paris) 12, 279 (1929)

[28] F. Bertaut, C. R. Acad. Sci. Paris 234, 1295 (1952).

[29] H. Forestier u. G. Guiot-Guillain, C. R. Acad. Sci. Paris 230, 1844 (1950)

[30] G. Guiot-Guillain u. H. Forestier, C. R. Acad. Sci. Paris 231, 1832 (1951).

[31] H. Forestier u. G. Guiot-Guillain, C. R. Acad. Sci. Paris 235, 48 (1952); G. Guiot-Guillain u. H. Forestier, ibid. 237, 1654 (1953)

[32] R. Pauthenet u. P. Blum, C. R. Acad. Sci. Paris 239, 33 (1954)

[33] L. Néel, C. R. Acad. Sci. Paris 239, 8 (1954).

[34] G. Guiot-Guillain, R. Pauthenet u. H. Forestier, C. R. Acad. Sci. Paris 239, 155 (1954)

[35] F. Bertaut u. F. Forrat, C. R. Acad. Sci. Paris 242, 382 (1956)

[36] R. Pauthenet, Ann. Phys. (Paris) 3, 424 (1958).

[37] R. Pauthenet, Ann. Phys. (Paris) 4, 710 (1952).

[38] H. A. Kramers, Physica I, 182 (1934).

\section{ZUSCHRIFTEN}

\section{Ein $\alpha$-Lactam des 2-Adamantylamins ${ }^{[* *]}$ \\ Von Erach R. Talaty, James P. Madden und Louis H. Stekoll ${ }^{[*]}$}

Es sind bereits mehrere $\alpha$-Lactame mit tert.-Alkylgruppen am Stickstoff dargestellt worden ${ }^{[1]}$, doch schlugen Versuche fehl, dort andere Typen von Alkylsubstituenten einzuführen; entweder entstanden dabei so reaktionsfähige $\alpha$-Lactame, daß sie nicht isoliert werden konnten, oder überhaupt keine ${ }^{[2]}$.

Wir konnten jetzt das bemerkenswert stabile $\alpha$-Lactam 1-(2-Adamantyl)-3-(1-adamantyl)aziridin-2-on (2a) darstellen, das eine sek.-Alkylgruppe in Position 1 trägt. Derartige Verbindungen könnten pharmazeutisches Interesse beanspruchen, da 1-Adamantylamin und seinen Derivaten antivirale Wirksamkeit zugeschrieben wird ${ }^{[3]}$.

Das Säurechlorid ( $\mathrm{Ia}$ ) wurde mit Brom in siedendem $\mathrm{CCl}_{4}$ behandelt, bis es vollständig in (1b) übergegangen

$\mathrm{R}^{1}-\mathrm{CHR}^{2}-\mathrm{CO}-\mathrm{R}^{3}(l)$

(a), $\mathrm{R}^{4}=1$-Adamantyl, $\mathrm{R}^{2}=\mathrm{H}, \mathrm{R}^{3}=\mathrm{Cl}$

(b), $\mathrm{R}^{1}=1$-Adamantyl, $\mathrm{R}^{2}=\mathrm{Br}, \mathrm{R}^{3}=\mathrm{Cl}$

(c), $\mathrm{R}^{2}=1$-Adamantyl, $\mathrm{R}^{2}=\mathrm{Br}, \mathrm{R}^{3}=\mathrm{NH}-2$-Adamantyl

[*] Prof. Dr. E.R. Talaty, J. P. Madden und L. H. Stekoll Department of Chemistry, Wichita State University Wichita, Kansas 67208 (USA)

[**] Diese Arbeit wurde vom Wichita State University Research Committee unterstützt. war. Die rohe Reaktionsmischung ergab mit 2-Adamantylamin in $71 \%$ Gesamtausbeute das Brom-amid (1c), $\mathrm{Fp}=237.5-240.0^{\circ} \mathrm{C}$ (Zers.) [NMR $\left(\mathrm{CDCl}_{3}, 60 \mathrm{MHz}\right.$, $\delta$-Werte in ppm gegen TMS $): 6.60(\mathrm{~N}-\mathrm{H} / \mathrm{br} . \mathrm{d}, \mathrm{J}=8 \mathrm{~Hz})$, $4.08(1 \mathrm{H} / \mathrm{s}), 4.08(1 \mathrm{H} / \mathrm{br} . \mathrm{d}, \mathrm{J}=8 \mathrm{~Hz}), 2.26-1.44(29 \mathrm{H} / \mathrm{m})$; Massenspektrum: $\left.\mathrm{m} / \mathrm{e}=407 / 405\left(\mathrm{M}^{+}\right), 326\left(\mathrm{M}^{+}-\mathrm{Br}\right)\right]$.

Die Reaktion von (lc) mit K-tert.-Butanolat in $\ddot{A}$ ther bei $0^{\circ} \mathrm{C}$ führte unter Ringschluß zum gewünschten $\alpha$-Lactam (2a), Fp $\approx 225^{\circ} \mathrm{C}$ (Zers.). Im Gegensatz zur Darstellung von $\alpha$-Lactamen wie $(2 b)^{[4]}$, bei der der Ringschluß durch überschüssige Base begünstigt wird, ohne daß sich dabei Nebenprodukte bilden, müssen bei der Synthese von (2a) die Mengenverhältnisse und Bedingungen sehr sorgfältig eingehalten werden. Die Isolierung von ( $2 a)$ ließ sich nur durch fraktionierende Kristallisation bei tiefer Temperatur vervollständigen. Die Struktur (2a) wird durch spektrale Daten gestützt [IR $\left(\mathrm{CCl}_{4}\right): v \approx 1850 \mathrm{~cm}^{-1} ; \mathrm{NMR}\left(\mathrm{CDCl}_{3}\right.$, $60 \mathrm{MHz}, \delta$-Werte in ppm gegen TMS): $2.53\left(\mathrm{H}^{3} / \mathrm{s}\right), 3.16$ $(1 \mathrm{H} /$ br.s $)$, ca. $2.4-1.4(29 \mathrm{H} / \mathrm{m}) ;$ Massenspektrum: $\mathrm{m} / \mathrm{e}$ $\left.=325\left(\mathrm{M}^{+}\right)\right]$.

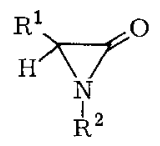

(a), $\mathrm{R}^{1}=1$-Adamantyl, $\mathrm{R}^{2}=2$-Adamantyl

(b), $\mathrm{R}^{\mathbf{t}}=\mathrm{R}^{2}=1$-Adamantyl

(c), $\mathrm{R}^{1}=\mathrm{C}_{6} \mathrm{H}_{5}, \mathrm{R}^{2}=\mathrm{C}\left(\mathrm{CH}_{3}\right)_{3}$

(d), $\mathrm{R}^{1}=\mathrm{R}^{2}=$ tert.-Alkyl 